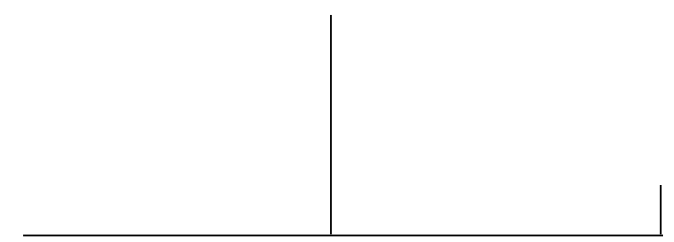

Rev. Latinoam. Psicopat. Fund., São Paulo, v. 15, n. 2, p. 309-322, junho 2012

\title{
O processo de trabalho na supervisão clínico-institucional nos Centros de Atenção Psicossocial (CAPS)*
}

\author{
Gilson Mafacioli da Silva \\ Carmem Lúcia Colomé Beck \\ Ana Cristina Costa de Figueiredo \\ Francine Cassol Prestes
}

\begin{abstract}
O objetivo deste estudo é descrever e analisar o processo de trabalho dos supervisores clínico-institucionais dos Centros de Atenção Psicossocial (CAPS) no Rio Grande do Sul, Brasil. Trata-se de uma pesquisa exploratório-descritiva, com abordagem qualitativa. Os sujeitos foram dez supervisores, e a técnica de coleta de dados foi feita a partir de entrevistas semiestruturadas. Os dados foram submetidos à análise temática. Como resultados identificaram-se ferramentas utilizadas pelo supervisor como suporte para sua prática no processo de trabalho, destacando-se: o mapeamento do processo de trabalho no CAPS, a experiência profissional na clínica e na saúde pública, com ênfase no SUS, o uso da escuta e da palavra, e a construção coletiva dos casos clínicos.
\end{abstract}

Palavras-chave: Processo de trabalho, supervisão clínico-institucional, Centros de Atenção Psicossocial, saúde mental

* Este artigo é parte integrante da dissertação de mestrado do Programa de pós-graduação em Psicologia, concluída na Universidade Federal de Santa Maria (RS, Br) no ano de 2010, intitulada "Processo de trabalho do supervisor clínico-institucional nos Centros de Atenção Psicossocial (CAPS)" na percepção dos supervisores. 


\section{Introdução}

No trabalho em saúde mental, percebe-se que são muitos os atravessamentos e desafios que surgem no cotidiano laboral, sejam eles pela grande demanda, pelas "urgências" reais e subjetivas dos sujeitos implicados, pelas limitações do trabalho em equipe, pelo desconhecimento e fragilidade da rede de saúde e das relações com os gestores, dentre outros.

Como dispositivos estratégicos na atenção psicossocial, os Centros de Atenção Psicossocial (CAPS) têm revelado estas realidades e demonstrado que as teorias e modelos prescritos de atendimento são insuficientes frente às demandas das relações diárias dos trabalhadores com o sofrimento e a singularidade deste tipo de atenção. Práticas e conceitos que pareciam sustentar o trabalho em saúde mental, a partir da formação acadêmica e de experiências no cotidiano laboral, exigem ser revistas antes de se tornarem estratégia terapêutica.

Assim, o trabalho nos CAPS pode ser um espaço de formação profissional que se constrói na prática e que se qualificará, caso os impasses presentes sejam revisados e superados.

Para isso, é fundamental a instauração de um ambiente democrático com estruturas e mecanismos institucionais que possibilitem o surgimento, desenvolvimento e manutenção de espaços intraequipes, em que se inclui o espaço da supervisão clínico-institucional como um dos que permite o florescimento de práticas fundadas na cooperação entre os saberes.

A proposta de supervisão clínico-institucional em implantação nos CAPS desde 2005 tem o objetivo de fortalecer e concretizar a política de atenção em saúde mental, a partir dos eixos norteadores da Reforma Psiquiátrica e do Sistema Único de Saúde (SUS) (Brasil, 2005).

O trabalho, sob esta perspectiva, proporciona aos profissionais procedimentos mais afinados de intervenção e de abordagens terapêuticas, facilitando uma escuta que permita uma melhor compreensão dos problemas. Compartilhar democraticamente os níveis de conhecimento teórico e de manejo técnico faz com que se desenvolva uma maior capacidade de tolerar a desigualdade e pode evitar que se estabeleçam aristocracias de saberes que podem fragmentar o grupo (Serrano Guerra, 2007).

Nesta perspectiva de reflexão, este estudo tem por objetivo descrever e analisar as ferramentas utilizadas no processo de trabalho dos supervisores clínico-institucionais dos CAPS no Rio Grande do Sul. 


\section{SAÚDE MENTAL}

\section{A função clínico-institucional da supervisão}

O Sistema Único de Saúde (SUS) instituído pelas Leis Federais 8.080/1990 e 8.142/1990 e os movimentos da Reforma Psiquiátrica traçaram novas diretrizes, promovendo modificações nas instituições, na assistência e no processo de trabalho em saúde.

No ano de 2002, a implantação dos CAPS foi regulamentada pela portaria 336/GM (Brasil, 2002), destacando-se que estes têm como papel fundamental e estratégico receber pacientes com grave sofrimento psíquico excluídos socialmente e organizar a rede de serviços de saúde mental do seu território, trabalho este a ser desenvolvido por equipe multiprofissional em uma perspectiva interdisciplinar.

Assim, o processo de trabalho na atenção psicossocial tem como uma de suas características ser sustentado por relações pessoais diretas de trabalhadores entre si, e com a população em geral, e mobilizar fortes implicações pessoais dos mesmos. Além disto, é preciso enfrentar problemas como um ambiente marcado pela falta de investimento, a precarização e a perda de direitos básicos do trabalho, o multiemprego, a deterioração da infraestrutura e a forte exigência de produtividade quantitativa que pode produzir sofrimento nos trabalhadores da saúde, neste contexto (Vasconcelos, 2008).

Para tanto, a supervisão clínico-institucional, financiada pelo Ministério da Saúde a partir da portaria GM 1.174, de 7/7/2005, é um dispositivo de formação permanente que deve sustentar a responsabilidade compartilhada da equipe, facilitar o diálogo para que as diferentes questões possam ser expostas, os casos e as situações sejam manejados com seus desafios e surpresas inerentes ao trabalho clínico na perspectiva da atenção psicossocial.

A supervisão atua de forma a sustentar a proposta de uma gestão partilhada da clínica, que auxilie os profissionais a enfrentarem os impasses institucionais frequentes no cotidiano do trabalho coletivo. A definição do espaço de cada trabalhador na equipe, mantendo a especificidade de seu ofício, mas quebrando a rigidez das especialidades, a pactuação de responsabilidades pelo fazer clínico mais do que pelos saberes em jogo, e a possibilidade de construção coletiva dos casos podem ser resultado deste dispositivo, permitindo avançar no trabalho em equipe e reduzir conflitos que podem comprometer seu funcionamento. (Figueiredo, 2008)

Dentre as tarefas características da supervisão (Brasil, 2009), destaca-se que ela seja clínica e institucional, levando-se em conta a integração da equipe de cuidado para possibilitar a construção de projetos terapêuticos que articulem os conceitos de sujeito, rede, território e autonomia.

Os supervisores podem ter formações teóricas e práticas diversas, desde que estejam abertos a exercê-las no cenário peculiar da rede pública, tendo como um dos desafios o de harmonizar as diversidades profissionais e teóricas (Brasil, 2009) 
A presença regular de um supervisor deve sustentar a ação dos profissionais nos serviços, conduzindo a uma produção permanente de conhecimento a partir da própria prática clínica. Assim, haveria a possibilidade do trabalho ter desdobramentos e, seu produto, um registro que inclua os impasses e dificuldades presentes neste percurso. Nesta perspectiva, o supervisor operaria como facilitador do trabalho, ou mesmo como garantia de trabalho e produção em equipe (Figueiredo, 2008).

\section{Sobre a pesquisa}

O presente estudo apresenta uma pesquisa exploratório-descritiva, com abordagem qualitativa. Os sujeitos de pesquisa foram supervisores clínicoinstitucionais do estado do Rio Grande do Sul/ Brasil, selecionados a partir do edital de Supervisão Clínico-Institucional de CAPS e Rede de Atenção Psicossocial do Ministério da Saúde.

O critério de inclusão dos participantes do estudo foi ser supervisor clínico-institucional em algum CAPS do Estado e ter sido selecionado desde 2005, quando iniciaram as atividades dos supervisores no RS. Foram excluídos do estudo os supervisores que não foram selecionados pelo Ministério da Saúde.

A seleção dos participantes se deu por meio de um sorteio dos supervisores que atenderam aos critérios de inclusão/exclusão do estudo, destacando-se também que se considerou a proporcionalidade das diferentes formações profissionais de graduação. Assim, participaram deste estudo dez supervisores clínico-institucionais de diferentes regiões do estado do RS, com formações teóricas e experiências profissionais diversas, atuantes em todas as modalidades de CAPS.

Foi utilizada como técnica de coleta de dados a entrevista semiestruturada ${ }^{1}$ e a observação não participante junto a um supervisor em seu processo de trabalho.

Após o sorteio dos participantes, foi realizado contato telefônico com os supervisores sorteados, a fim de explicar o objetivo da pesquisa, realizar o convite

1. Antes da entrevista, os participantes foram informados sobre os objetivos do estudo e do caráter voluntário de sua participação, sendo solicitado que lessem o Termo de Consentimento e, em caso de concordância com os termos expostos, assinassem o documento, em duas vias, sendo que uma foi fornecida ao participante e outra ficou de posse do pesquisador. Nesse momento, também foi solicitada a autorização para gravação das entrevistas no intuito de assegurar a fidedignidade das respostas.

Rev. Latinoam. Psicopat. Fund., São Paulo, v. 15, n. 2, p. 309-322, junho 2012 


\section{SAÚDE MENTAL}

para participação e, mediante aceite, agendar data e local para a realização das entrevistas. Não houve recusas entre os supervisores contatados e as entrevistas foram realizadas no período de abril a junho de 2010 .

As entrevistas foram transcritas e lidas para apreensão inicial do conteúdo, sendo que a leitura do material permitiu apreender o conteúdo manifesto e agrupar os fragmentos que se repetiam e/ou possuíam semelhança semântica nos diferentes depoimentos. Posteriormente, procedeu-se a categorização dos elementos, completando-se as três etapas de análise: pré-análise; exploração do material e tratamento dos resultados obtidos e interpretação.

Os dados foram submetidos à análise temática, uma das modalidades da análise de conteúdo, fundamentada na regularidade das afirmações denotando, dessa forma, estruturas de relevância, valores de referência e comportamentos presentes ou subjacentes nos depoimentos (Minayo, 2000). Assim sendo, foram construídas duas categorias, quais sejam: ferramentas utilizadas como apoio ao trabalho do supervisor e ferramentas utilizadas no desenvolvimento do trabalho junto à equipe.

\section{A experiência do Rio Grande do Sul em questão}

Evidenciou-se que os supervisores utilizaram algumas ferramentas de trabalho na implementação das propostas de supervisão. Dentre os dados emergidos neste estudo, foram organizadas duas categorias: ferramentas utilizadas como apoio ao trabalho do supervisor e ferramentas utilizadas no desenvolvimento do trabalho junto à equipe.

No que se refere às ferramentas utilizadas como apoio ao trabalho do supervisor, primeira categoria, identificou-se a construção de um mapeamento do processo de trabalho; a experiência do dia a dia na saúde coletiva e a experiência profissional e pessoal.

A 'construção de um mapeamento do processo de trabalho no CAPS' antes de iniciar a atividade foi relatada como essencial por alguns supervisores. Assim, para o início das atividades de supervisão alguns dos entrevistados perceberam a necessidade de coletar dados do serviço, entre eles, o número de funcionários, as funções, o tipo de vínculo empregatício, o número de usuários, os tipos de atendimentos, as relações com o usuário e seus familiares, com a equipe, com gestores e com a rede.

Este levantamento inicial servia para "desnudar" as nuanças das práticas do serviço para posterior elaboração de um projeto de trabalho e de encontros pré-programados. Os fragmentos abaixo atribuem sentidos a esta investigação inicial: 
[...] a coleta destes dados possibilita estabelecer diálogos e, dentro da perspectiva do SUS, fazer girar todos eles [...].

A 'experiência cotidiana na saúde coletiva' também foi mencionada como importante para os supervisores no sentido de ter o conhecimento das demandas apresentadas no SUS para além dos consultórios particulares. A seguir, identifica-se a relevância dada pelos supervisores ao conhecimento da realidade do SUS e a experiência do trabalho em equipe para a prática deste ofício.

[...] para ser supervisor tem que ter experiência de trabalho institucional e SUS, não necessariamente em CAPS, o próprio trabalho em hospital, na universidade, enfim, porque é de relações que se trata, de algum tipo específico de laço social que está ali fundado e que, apenas o saber acadêmico não vai dizer o que é [...].

A 'experiência profissional e pessoal' também é utilizada no processo de trabalho da supervisão. Campos e Garcia (2007) relatam que, na ausência de parâmetros consistentes, cabe ao supervisor construir seu próprio modo de atuar a partir da prática, no qual, inevitavelmente, vai imprimir traços da sua própria trajetória, de sua vivência de quando foi supervisionado.

A 'experiência profissional ou pessoal como instrumento de trabalho', não foi usada de forma isolada, mas associada à formação específica de cada profissional. Sob esta ótica, não seria uma recusa dos conhecimentos científicos adquiridos, mas o reconhecimento de que o processo de convívio e as relações que se estabelecem nestas experiências contribuem para a construção do conhecimento.

A maioria dos sujeitos afirmou que conduz o processo de supervisão com uma postura mais flexível, fundamentada no momento em que as equipes se encontravam, como revela a fala a seguir.

[...] eu costumo ter um planejamento, não rígido, nas direções de trabalho para cada encontro, possibilidades de propostas, de dispositivos para facilitar, que circule a palavra entre a equipe. Mas normalmente, como que eu acho que deve acontecer em todas as situações, sempre vai ser diferente do que tu planejas, seja por uma administração do tempo, seja porque tu precisas assinalar mais um tópico do que outro [...]

Além disto, foi considerado que intervenções em ato podem ser necessárias. Isto dependerá da confiança, do vínculo e da transferência existentes. Fazer a devolução do que foi trazido pela equipe e daquilo que pareceu ser indispensável das falas, também foi proposto por alguns supervisores.

Figueiredo (2005) refere que no modo de organização coletiva:

As especialidades sofrem uma "implosão", devendo todos os participantes ter a palavra, seja em que momento for. A hierarquia dá lugar a uma autorização

Rev. Latinoam. Psicopat. Fund., São Paulo, v. 15, n. 2, p. 309-322, junho 2012 


\section{SAÚDE MENTAL}

difusa e sempre questionável de um profissional por outro. Tudo pode ser discutido e o que prevalece é o confronto de "igualdades", em que cada diferença pode ser tomada como desavença ou ameaça ao equilíbrio harmonioso do todo, no qual se sustenta a equipe. Aqui, a equipe se sustenta na lógica do "todo" [...] (p. 44)

Esta situação é percebida pelos entrevistados como possibilidade de intervenção e que leva a desdobramentos na direção do processo de trabalho coletivo nos CAPS, conforme ilustrado a seguir:

[...] a presença do supervisor pode autorizar as pessoas a falarem e a disputarem certas concepções, permitindo a construção de um entressaberes, que é um saber a mais, que opere cuidado, saúde e contribua na produção de um saber do próprio usuário sobre si [...].

Finalizando esta discussão, para alguns pesquisados, é necessário um momento de fechamento a cada encontro, no qual as pessoas possam dizer como se sentiram, uma vez que, neste momento, algumas considerações podem emergir e servirem para monitorar e avaliar trabalho de supervisão.

No que se refere à segunda categoria, ferramentas utilizadas no desenvolvimento do trabalho junto à equipe identificaram-se a escuta e a palavra; a construção coletiva dos casos clínicos; a leitura de textos, a participação de convidados em temas específicos, a problematização das situações do cotidiano das equipes dos CAPS e a transferência de trabalho.

A 'escuta e a palavra' como recursos operativos para a supervisão foram citadas por todos os supervisores como ferramentas imprescindíveis. Elas possibilitam a circulação da complexidade dos discursos nas reuniões de equipe, considerando-se uma escuta livre, aberta, desprovida de preceitos morais, vinda de alguém que não é detentor da verdade. Que "descole e desloque" os profissionais das prescrições preconcebidas de funcionamento dos serviços e que os retire de uma posição de alienação, de não interrogação.

[...] A supervisão é especialmente uma escuta do grupo que trabalha, cada um na sua especificidade [...], na sua linguagem, no seu imaginário e que é um grupo heterogêneo e que nem sempre entende o sujeito da mesma forma, o mesmo fenômeno da mesma forma e, justamente por isso, precisa se escutar entre si. [...]

Neste sentido, Oliveira, Palma e Veronese (2009) propõem como reflexão que "a escuta daquilo que retorna como efeito de um discurso automatizado, circunscrito na impessoalidade, pode indicar palavras que possam estabelecer uma dimensão subjetiva, ou seja, que sirvam como suporte para a construção de um arranjo singular, no lugar de uma fala automatizada e condescendente" (p. 1351). Para estas autoras, escutar e apontar para uma palavra que indique sua constituição subjetiva parece ser um recurso fundamental. 
A 'construção coletiva dos casos clínicos', segundo os supervisores, pode servir para avaliar o funcionamento da equipe e a implicação de cada um no trabalho com o usuário. Casos que envolvam toda a equipe, nos quais haja dúvidas quanto aos encaminhamentos, podem expor os atravessamentos de uns com os outros, do quanto um propõe algo e o outro desconsidera.

Evidenciam-se questões de um profissional com o outro, diferenças importantes que dificultam o trabalho na equipe. A partir de algo que não anda bem na direção do tratamento com um ou outro usuário, podem se revelar pontos de dissociação ou de rupturas da equipe. Poderia se considerar, então, um espaço de cuidado com o serviço, com a política e com o usuário. Caberia, então, ao supervisor, sustentar a dimensão clínica quando todos se perdem no político, pontuando estes momentos.

Outro instrumento descrito pelos supervisores foi a 'leitura de textos sugeridos pelo supervisor ou pelas equipes'. Estes tinham como objetivo embasar as supervisões, ampliando o olhar de alguns problemas e ajudar a equipe a encontrar possíveis soluções.

Alguns textos foram considerados obrigatórios como, por exemplo, os que abordam as leis do SUS e a Reforma Psiquiátrica. Alguns supervisores sugeriram leituras específicas e complexas que funcionaram como obstáculos para a equipe e não favoreceram o sucesso desta proposta. A fala abaixo contextualiza tais proposições:

[...] a supervisão tem que permitir que a equipe reconheça o momento em que se encontra, que tipo de construção, que tipo de assimilação dos conceitos que tem e onde eles podem buscar aprofundar. Permitir um aprofundamento desde que seja uma questão para eles e não algo que é somente imposto [...].

Dois entrevistados, ao identificarem dificuldades com temas específicos para trabalhar determinada necessidade do grupo, utilizaram como estratégia o convite a outro profissional com mais experiência nestas questões, o que teve resultado positivo junto à equipe.

[...] existia a ideia de se convidar algumas pessoas para fazer alguns seminários

[...] não é porque tu fazes trabalho de supervisão, que tu vais dar conta do todo $[\ldots]$

A 'problematização das situações do cotidiano das equipes dos CAPS' foi referida, em várias entrevistas, como forma de trabalho das supervisões. Esta metodologia teria como objetivo propiciar o diálogo entre os participantes e as diferentes concepções e campos de trabalho, abrindo espaço para novas reflexões e novos acordos. Problematizar implicava em fazer com que a equipe interrogasse, repensasse e questionasse suas condutas, ou melhor, a sua própria clínica. Nesta 


\section{SAÚDE MENTAL}

perspectiva, ao iniciar por um problema, encadearia outro e isto ocorreria sucessivamente.

A partir desta análise, a supervisão poderia ser um espaço não só de resolução, mas também de construção de problemas. Elegia-se uma questão trazida por alguém da equipe e havia o detalhamento da mesma, a partir de perguntas colocadas à pessoa que a enunciou. A partir das respostas e das próprias perguntas que os outros integrantes da equipe faziam, tinha-se aquilo que iria ser trabalhado e que podia não coincidir com o que foi enunciado. Por esta concepção haveria um hiato entre o que foi dito que queria ser trabalhado e o que efetivamente apareceu como emergente, como uma necessidade do grupo.

Tal argumento de ação permitiria que os trabalhadores olhassem objetivamente para a realidade do seu trabalho. Haveria um estímulo à construção ou à reformulação das hipóteses colocadas na discussão, substituindo-as por outras mais próximas da realidade, a partir do olhar de vários trabalhadores. A equipe pode se referenciar em si, ou seja, no que ela já tem de conhecimento acumulado para que não se construa uma relação de dependência com o supervisor clínico-institucional.

[...] Problematizar permite emergir como é que a equipe está entre ela, com o usuário, com a rede [...] Diferentes concepções podem ser conjugadas numa mesma equipe, sem homogeneizar o conhecimento. Muitas vezes, as pessoas não têm o significado claro do que sabem, sobre o que pensam ou o porquê pensam de determinada maneira $[\ldots]$

Entende-se que a problematização como processo educativo e pedagógico, produz um trabalho de educação permanente que não impõe os ideais do supervisor em detrimento do desejo e do conhecimento dos trabalhadores nas equipes.

Em relação a este fato, na concepção de Conte, Plein e Silveira (2009), a educação permanente em saúde é uma política e uma prática que inverte a lógica de lugar de objeto que ocupam os trabalhadores. Ou seja, retira os trabalhadores da lógica de receber conteúdos e de reprodução de um saber.

Foi destacado também que a garantia do trabalho e a direção clínica construída em equipe são condições para que o dispositivo de supervisão produza efeitos (Figueiredo, 2008). Um importante balizador para o trabalho em equipe, com suporte na supervisão sob referência da psicanálise, é a "transferência de trabalho". Nesta perspectiva:

[...] a transferência de trabalho é concebida a partir do próprio conceito de transferência, central no tratamento psicanalítico, mas dessa vez como um instrumento do trabalho entre pares. Seria a condição do estabelecimento de um laço produtivo entre pares visando, por um lado, o fazer clínico e, por outro, a produção de saber que lhe é consequente. A transferência que deve operar no trabalho em equipe deve ser norteada pelo fato de que há um objetivo comum às 
diferentes profissões, que é uma determinada concepção da clínica pautada no sujeito.

Validando o exposto, estabelecer transferências de trabalho em uma equipe, para Ribeiro (2009, p. 18), significaria que todos partiriam de uma posição de não saber. Este seria o "em comum" da equipe. O autor pontua que "cada um só pode falar a partir de sua trajetória pessoal e profissional, mas que nesta experiência ninguém falaria de sua especialidade e, menos ainda, autorizado por ela". Haveria um deslocamento da suposição do saber, da equipe, para os sujeitos por ela atendidos (ou, neste caso, para os supervisionados). A singularidade de cada caso (situação) é que irá orientar a ação da equipe/supervisor.

No decorrer dos encontros e com um vínculo ou transferência de trabalho estabelecido, as equipes se permitem ocupar o espaço de supervisão para trabalhar as dificuldades existentes entre si e com a gestão. A presença do supervisor parece viabilizar e principalmente dar suporte a esse diálogo.

\section{Considerações finais}

O trabalho na saúde mental é um campo de incertezas e não de generalizações, pois parte de sujeitos e de suas singularidades. Sob este prisma, os supervisores pesquisados neste estudo evidenciam a importância da construção de espaços para a problematização e discussão do processo de trabalho em saúde.

Neste estudo, foram apresentadas as ferramentas de trabalho do supervisor clínico-institucional enredado entre os fenômenos clínicos e políticos projetados no campo da saúde coletiva, o qual tem se tornado palco de várias discussões e conflitos.

Reconhece-se que a mudança da lógica de trabalho, proposta pela política de saúde, não é tarefa fácil de ser implementada pelas equipes nos CAPS, pois não ocorre automaticamente, como determinam as diretrizes, e recebe críticas quando assumida como um fazer prescrito. Acredita-se que ela deve ser trabalhada junto às equipes, no espaço proposto da supervisão, como um processo de formação permanente que opere novos sentidos e que permita a reflexão e a análise crítica sobre o próprio trabalho. Destaca-se que a política pode amparar as mudanças dos paradigmas vigentes, contribuindo com contornos à construção de novos conhecimentos.

\section{Referências}

Brasil, Ministério da Saúde. Portaria 336/GM de 19 de fevereiro de 2002. 


\section{SAÚDE MENTAL}

Brasil, Ministério da Saúde. Portaria 1174/GM de 7 de julho de 2005.

Brasil, Ministério da Saúde. O ofício da supervisão e sua importância para a rede de saúde mental do SUS. Portal da Saúde - www.saude.gov.br-saudemental, 2007a.

Brasil, Ministério da Saúde. Portaria 1996. Dispõe sobre as diretrizes da implantação da Política Nacional de Educação Permanente em Saúde. 20 de agosto de 2007c.

CAmpos, C.; Garcia, J. Contribuições para a supervisão dos programas sociais com foco na família. Rev. katálysis [online], v. 10, n. 1, p. 95-104, 2007.

Conte, M.; Plein, F.B.; Silveira, M. Saúde Coletiva, psicanálise e educação permanente em saúde. Boletim da Saúde. Escola de Saúde Pública, RS, v. 23, n. 2, p. 130-138, jul/dez.2009.

Figueiredo, A.C. Uma proposta da psicanálise para o trabalho em equipe na atenção psicossocial. Mental [online], v. 3, n. 5, p. 43-55, 2005 [citado 2010-10-31]. Disponível em: <http://pepsic.bvsalud.org/scielo.php?script=sci_arttext\&pid=S1679>.

Duas ou três coisas que aprendemos sobre a função da supervisão na atenção psicossocial, em 12 anos de CAPS na cidade do Rio de Janeiro. Revista da Escola de Saúde Mental, SMS-Rio, ano 1, n. 1, p. 63-69, dez.2008.

Minayo, M.C. de S. O desafio do conhecimento: pesquisa qualitativa em saúde. 7. ed. São Paulo: Hucitec; Rio de Janeiro: Abrasco, 2000.

Oliveira, I.M.A,; Veronese, L.G.; Palma, C.M.S. A servidão mais que voluntária: dispositivos burocráticos em instituição de saúde mental. Rev. Mal-Estar Subj., v. 9, n. 4, p. 1343-1354, dez.2009.

Ribeiro, E.E.M. A psicanálise nas instituições: clínica e política. Boletim da Saúde, Escola de Saúde Pública, RS., v. 23, n. 2, p. 15-21, jul/dez.2009.

SERrano Guerra, E. Los procesos de formación dentro de los equipos interdisciplinares de salud mental. Rev. Asoc. Esp. Neuropsiq., Madrid, n. 86, jun. 2003. Disponível em: <http://scielo.isciii.es/scielo.php?script=sci_arttext\&pid=S0211$57352003000200001 \& \operatorname{lng}=\mathrm{es} \& n r m=$ iso > . Acessado em 28 nov. 2009>.

Vasconcelos, E.M. Abordagens psicossociais. V.1. História, Teoria e Trabalho no Campo. São Paulo: Hucitec, 2008.

\section{Resumos}

(The process of working in clinical-institutional supervision at Brazilian psychosocial treatment centers, referred to as CAPS)

The aim of this study is to describe and analyze the working process of clinical and institutional supervisors at Psychosocial Treatment Centers (CAPS) in the state of 
Rio Grande do Sul, Brazil. This is an exploratory and descriptive study based on a qualitative approach. The subjects were ten supervisors and the data was gathered through semi-structured interviews. The data was submitted to theme analysis. The results presented instruments used by supervisors as they carry out their work, such as mapping the working processes at the centers, as well as their clinical experience and broader professional experience in the Brazilian public health system, listening to patients as they talk, and the collective construction of clinical cases.

Key words: Working process, clinical and institutional supervision, psychosocial treatment center, mental health

(Le processus de travail de la supervision clinico-institutionnel des Centres de Soins Psychosociaux - CAPS)

Le but de cet étude est de décrire et d'analyser le processus de travail des superviseurs clinico-institutionnels des Centres de Soins Psychosociaux (CAPS) du Rio Grande do Sul, Brésil. Il s'agit d'une recherche exploratoire et descriptive, d'approche qualitative. Les sujets étaient composés de dix superviseurs et les données ont été obtenues par moyen d'entretiens semi-directifs. Les données ont ensuite été soumises à une analyse thématique. Les résultats portaient sur les outils utilisés par les superviseurs comme support de travail aux Centres, soit: la cartographie du processus de travail aux Centres, l'expérience professionnelle acquise en clinique et dans le système de santé publique brésilien (SUS), l'usage de l'écoute et de la parole, ainsi que la construction collective des cas cliniques.

Mots clés: Processus de travail, supervision clinico-institutionnel, Centre de Soins Psychosociaux, santé mental

(El proceso de trabajo de la supervisión clínico-institucional en los Centros de Atención Psicosocial - CAPS)

El objetivo de este estudio fue describir y analizar el proceso de trabajo de los supervisores clínico-institucionales de los Centros de Atención Psicosocial (CAPS) en Río Grande del Sur, Brasil. Se trata de una pesquisa de carácter exploratoria y descriptiva, con abordaje cualitativa. Los sujetos fueron diez supervisores, y la técnica de colecta de datos utilizada fueron entrevistas semi-estructuradas. Los datos fueron examinados por el método del análisis temático. Como resultados se identificaron herramientas utilizadas por el supervisor como soporte para su práctica en el proceso de trabajo, destacándose: el delineamiento del proceso de trabajo en el CAPS, la experiencia profesional en la clínica y en la salud pública con énfasis en el SUS, el uso de la escucha y de la palabra, y la construcción colectiva de casos clínicos.

Palabras claves: Proceso de trabajo, supervisión clínico-institucional, Centro de Atención Psicosocial, salud mental 


\section{SAÚDE MENTAL}

(Der Arbeitsprozess in der Verwaltung klinischer Einrichtungen in den Centros de Atenção Psicossocial (CAPS) (Zentren für Psychosoziale Behandlung in Brasilien)

Ziel dieser Studie ist die Beschreibung und Untersuchung des Arbeitsprozesses des klinischen Aufsichtspersonals in den Centros de Atenção Psicossocial (CAPS) (Zentren für Psychosoziale Behandlung in Brasilien). Es ist eine forschende und beschreibende Studie mit qualitativem Ansatz. Die Studie wurde an zehn Führungspersonen durchgeführt und die Datenerhebungstechnik basierte auf teilweise vorstrukturierten Interviews. Die zusammengetragenen Daten wurden thematisch untersucht. Daraus ergab sich die Identifizierung einiger Instrumente der Leiter zur Unterstützung ihrer Praxis in Arbeitsprozessen, insbesondere: Die Erstellung einer Übersicht der Arbeitsprozesse im CAPS, die Berufserfahrung in der Klinik und im öffentlichen Gesundheitswesen, insbesondere des SUS (Sistema Único de Saúde - brasilianisches Einheitliches Gesundheitssystem), das Hören, die Anwendung von Wörtern sowie die Erstellung kollektiver klinischer Daten.

Schlüsselwörter: Arbeitsprozess, klinische Verwaltung, Centro de Atenção Psicossocial (Zentrum für Psychosoziale Behandlung in Brasilien), geistige Gesundheit

Citação/Citation: Silva, G.M. DA; Beck, C.L.C.; Figueiredo, A.C.C.; Prestes, F.C. O processo de trabalho na supervisão clínico-institucional nos Centros de Atenção Psicossocial (CAPS). Revista Latinoamericana de Psicopatologia Fundamental, São Paulo, v. 15, n. 2, p. 309-322, jun.2012.

Editor do artigo/Editor: Profa. Dra. Ana Cristina Costa de Figueiredo

Recebido/Received: 2.9.2011 / 9.2.2011 Aceito/Accepted: 15.12.2011 / 12.15.2011

Copyright: () 2009 Associação Universitária de Pesquisa em Psicopatologia Fundamental/ University Association for Research in Fundamental Psychopathology. Este é um artigo de livre acesso, que permite uso irrestrito, distribuição e reprodução em qualquer meio, desde que $\mathrm{o}$ autor e a fonte sejam citados / This is an open-access article, which permits unrestricted use, distribution, and reproduction in any medium, provided the original author and source are credited.

Financiamento/Funding: Os autores declaram não ter sido financiados ou apoiados / The authors have no support or funding to report.

Conflito de interesses/Conflict of interest: Os autores declaram que não há conflito de interesses / The authors declare that has no conflict of interest.

\section{Gilson Mafacioli da Silva}

Médico Psiquiatra; Especialista em Saúde Coletiva na atenção a usuários de álcool e outras drogas pela Pontifícia Universidade Católica do Rio Grande do Sul - PUC-RS (Porto Alegre, RS, Br); Mestre em Psicologia pela Universidade Federal de Santa Maria - UFSM (Santa Maria, 


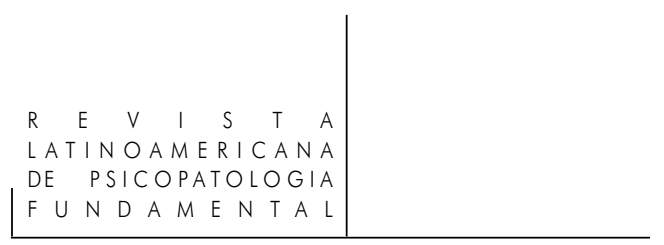

RS, Br); Membro do Grupo de Pesquisa Trabalho, Saúde, Educação e Enfermagem da Universidade Federal de Santa Maria - UFSM (Santa Maria, RS, Br).

Rua Major Duarte, 240/401

97050-460 Santa Maria, RS, Br

Fone: (55) 9996-6410

e-mail: gilsonmafa@terra.com.br

\section{Carmem lúcia Colomé Beck}

Enfermeira; Prof ${ }^{a} \mathrm{D}^{\text {ra }}$ do Depto. de Enfermagem da Universidade Federal de Santa Maria UFSM (Santa Maria, RS, Br); Membro do Grupo de Pesquisa Trabalho, Saúde, Educação e Enfermagem da Universidade Federal de Santa Maria - UFSM (Santa Maria, RS, Br); Docente do Programa de Pós-Graduação da Psicologia e da Enfermagem da Universidade Federal de Santa Maria - UFSM (Santa Maria, RS, Br).

Rua Estácio Mariense de Lemos, 154 - Bairro Patronato

97020-150 Santa Maria, RS, Br

Fone: (55) 9674-5401; (55) 3307-8230

e-mail: carmembeck@gmail.com

\section{Ana Cristina Costa de Figueiredo}

Psicóloga; Mestrado em Psicologia Clínica pela Pontifícia Universidade Católica do Rio de Janeiro - PUC-Rio (Rio de Janeiro, RJ, Br); Doutorado em Saúde Coletiva pelo Instituto de Medicina Social da Universidade do Estado do Rio de Janeiro - UERJ (Rio de Janeiro, RJ, Br); Professora Associada do IPUB - Instituto de Psiquiatria da Universidade Federal do Rio de Janeiro - UFRJ (Rio de Janeiro, RJ, Br); Diretora de Ensino dessa instituição e Professora Colaboradora do Programa de Pós-graduação em Psicanálise da Universidade do Estado do Rio de Janeiro - UERJ (Rio de Janeiro, RJ, Br).

Universidade Federal do Rio de Janeiro

Instituto de Psiquiatria - Programa de Psiquiatria

Av. Venceslau Brás, 71 - Botafogo

22290-140 Rio de Janeiro, RJ, Br

Fone: (21) 2295-2549

e-mail: anacrisfigueiredo@gmail.com

\section{Francine Cassol Prestes}

Enfermeira; Mestranda do Programa de Pós-Graduação na Universidade Federal de Santa Maria - UFSM (Santa Maria, RS, Br); Membro do Grupo de Pesquisa Trabalho, Saúde, Educação e Enfermagem da Universidade Federal de Santa Maria - UFSM (Santa Maria, RS, Br).

Rua Tuiuti, 520/202 - Centro

97420-000 Santa Maria, RS, Br

Fone: (55) 9962-2401

e-mail: francinecassol@gmail.com 